\title{
INDIRECT PROOF AND INVERSIONS OF SYLLOGISMS
}

\author{
ROY DYCKHOFF
}

\begin{abstract}
By considering the new notion of the inverses of syllogisms such as Barbara and Celarent, we show how the rule of Indirect Proof, in the form (no multiple or vacuous discharges) used by Aristotle, may be dispensed with, in a system comprising four basic rules of subalternation or conversion and six basic syllogisms.
\end{abstract}

$\S 1$. Introduction. Aristotle's theory of deduction includes a rule $I P$ of Indirect Proof; one major result about it is in von Plato's work $[12,13,11]$, that the sub-deductions of a deduction may be permuted so that the rule only appears at the last step of a deduction. But, it is not even clear that modern scholars are unanimous about what exactly the rule says: are multiple or vacuous discharges permitted, as in [3, 4], or forbidden, as in $[7,12,13,11]$ ? We use the latter approach, forbidding multiple or vacuous discharge. Several papers have shown how the rule may be dispensed with: a simple approach using ecthesis (the use of fresh individual variables), as in $[8,5]$ or a more far-reaching approach, as in [9]. Another obvious approach is just to add all the 24 valid syllogisms as basic rules; this seems to be cheating.

By enlarging (to four) the number of simple rules of subordination or conversion, and then adding a minimal set of six syllogisms, we are able to show that the rule $I P$ is admissible. This set of syllogisms has to include Barbara, Baroco and Bocardo; otherwise, there is some flexibility, but the purest version adds Celarent, Disamis and Festino. This choice is explained and justified by the new notion of the inverses of a syllogism and by an elementary study of the relationships, between the 24 valid syllogisms, determined by the four simple rules.

§2. Aristotle's formal system. In this section we spell out a simplified view of the formal system of Aristotle: it has some interesting features, which turn out to assist proof-theoretical analysis not done until recently (e.g. $[12,13,11])$.

Received by the editors June 2018 .

1991 Mathematics Subject Classification. 03F99.

Key words and phrases. Deduction, syllogism, indirect proof. 
2.1. Terms and propositions. Terms will be denoted by letters $P$, $Q, R, S$, etc. As examples we think of various concepts like "horse", "human", "unicorn", "cat", "bearded", "tall", etc. Individuals like Socrates can be regarded as denoted by terms, by considering the concept or property of being Socrates. Aristotle avoided non-denoting terms, such as "unicorn".

Simple "propositions" (aka "statements"), $A, B, C, D$, etc., about such terms may be formed, such as that "Some horse is bearded", "Every cat is human", "No bearded thing is tall", "Some cat is not tall", etc. Aristotle's expression was more like (for the positive statements) "beardedness holds of some horse", "humanity holds of every cat", etc., i.e. putting the predicate before the subject (as in modern formal treatments of quantifier-free monadic logic). We shall use the form closest to English grammar, which has, at least for the positive statements, the subject before the predicate.

Propositions are divided into

1. the Affirmative (aka Positive) Propositions of the form

(a) "P holds of every S", "All S are P", "Every S is P", "P belongs to every S", "P holds of all S";

(b) "P holds of some S", "P belongs to some S", "Some S is P";

2. the Negative Propositions of the form

(a) "P does not hold of any $\mathrm{S}$ ", "P holds of no S", "No S is P";

(b) "P does not hold of all S", "P holds of not all S, "Not all S are $\mathrm{P}$ ", "Some $\mathrm{S}$ is not $\mathrm{P}$ ", "P does not hold of some $\mathrm{S}$ ".

Those of form 1 (a) we will write (following von Plato) ${ }^{1}$ as $\Pi^{+}(S, P)$; those of form $1(\mathrm{~b})$ we will write as $\Sigma^{+}(S, P)$. Those of form $2(\mathrm{a})$ we will write as $\Pi^{-}(S, P)$; those of form $2(\mathrm{~b})$ we will write as $\Sigma^{-}(S, P)$.

The Universal ones are of the form $\Pi^{+}(S, P)$ or $\Pi^{-}(S, P)$; the Existential ones are of the form $\Sigma^{+}(S, P)$ or $\Sigma^{-}(S, P)$. Those of the form $\Pi^{+}(S, P)$ or $\Sigma^{+}(S, P)$ are Positive; the others are Negative.

In each case, $S$ is said to be the Subject and $P$ the Predicate of the proposition. There is then a simple and obvious notion of interpretation using non-empty sets.

Each proposition has a contradictory: $\Pi^{+}(S, P)$ and $\Sigma^{-}(S, P)$ are contradictory, in the sense that (using interpretations) each is "equivalent to the negation of" the other: if one is false the other is true, and vice-versa. Likewise, $\Pi^{-}(S, P)$ and $\Sigma^{+}(S, P)$ are contradictory in the same sense.

\footnotetext{
1 Other notations are $A(S, P), I(S, P), E(S, P)$ and $O(S, P) ; S a P, S i P, S e P$ and $S o P$; the copulae $A, S, N$ and $\$$ (for "All", "Some", "No" and "Not Some") are also used (but then it is best not to use $S$ for subjects). Our choice (following [12]) makes immediately clear which is universal, which is positive, etc; we have no need to rely on mnemonics such as that $e$ and $o$ are parts of the Latin word Nego (I deny).
} 
We will write $A^{*}$ for the contradictory (as thus defined) of the proposition $A$. (Note that $\Pi^{-}(P, S)$ is equivalent to $\Pi^{-}(S, P)$, so the notion of being "the contradictory" could be weakened.) Then $A^{* *}=A$.

Furthermore, $\Pi^{+}(S, P)$ and $\Pi^{-}(S, P)$ are contraries in the sense that they cannot both be true.

2.2. Deductions and syllogisms. The first task is to choose what kind of "collection" should form a collection of assumptions. We are mindful of the variety [10] of formal systems that cover, for example, classical logic and that vary only in respect to such choices, e.g. between list, multiset or set. On the one hand, Corcoran [3] proposes that (for Aristotle's logic) it is a set. On the other hand, Smiley [7, p. 141] talks first of "set of wffs", but then qualifies this as follows:

"..., we shall want to construe the notion of a set of wffs so as to take account of their multiplicity of occurrence. This is most easily done by taking 'set of wffs' always to mean 'set of occurrences of wffs', and counting the number of members accordingly. For example, $P, P, Q$ will be a different set from $P, Q$, and the former will have three members while the latter only has two."

Smiley thus has in mind the notion of a multiset of wffs. He nevertheless goes on to define logical consequence (of a wff $Q$ from a set $X$ of wffs) by ignoring the difference between multisets and sets.

Von Plato $[12,13,11]$ resolves the issue by working entirely in a natural deduction setting, and presenting a rule, "the scheme of indirect proof", allowing multiple and vacuous discharge of an assumption, then making clear that "it is typical of Aristotle's proofs that an assumption closed in indirect proof occurs just once" and then just using this restricted form, clearly enforced by the use of multisets rather than sets. We shall take this latter form to be Aristotle's Principle of Indirect Proof.

Deductions are of a proposition $A$ from a multiset $\Gamma$ of assumptions. Multisets are combined using sum rather than union. The Rules of Inference $2^{2}$ are as follows:

A1: If we have deduced $\Pi^{+}(P, S)$, then we may deduce $\Sigma^{+}(S, P)$;

A2: If we have deduced $\Pi^{-}(P, S)$, then we may deduce (by conversion) $\Pi^{-}(S, P)$;

A3: If we have deduced $\Pi^{+}(M, P)$ and $\Pi^{+}(S, M)$, then we may deduce (by syllogism) $\Pi^{+}(S, P)$;

A4: If we have deduced $\Pi^{-}(M, P)$ and $\Pi^{+}(S, M)$, then we may deduce (by syllogism) $\Pi^{-}(S, P)$;

2 The order in which we present the premisses in rules $A 3$ and $A 4$ is to ensure that, when $\Pi^{-}$is replaced by $E$ and $\Pi^{+}$by $A$, the letters appear in the order that appears in the traditional names $b A r b A r A$ and $c E l A r E n t$ for these rules. This has the effect that the Middle Term, $M$, no longer appears in the middle. But, as it is in the middle for only $25 \%$ of the valid syllogisms, this is not a difficulty. 
IP: If we have deduced $B$ from $A^{*}$ and also have deduced $B^{*}$, then we may combine the two deductions, remove (i.e. discharge) the single assumption of $A^{*}$ and thus form a deduction of $A$ (from the multiset sum of the two multisets of undischarged assumptions).

$I P$ is a rule of Indirect Proof. Note that the discharged occurrence of the assumption $A^{*}$ is in the first premiss. Neither vacuous nor multiple discharge is allowed. Other occurrences (if any) of $A^{*}$ are left undischarged. The other rules are called Rules of Direct Proof.

There is no rule of Weakening: the logic is substructural.

Rules $A 3$ and $A 4$ are basic syllogisms, known respectively since mediaeval times as Barbara and Celarent. We present them in tree style:

$$
\frac{\Pi^{+}(M, P) \quad \Pi^{+}(S, M)}{\Pi^{+}(S, P)} \text { Bar } \quad \frac{\Pi^{-}(M, P) \quad \Pi^{+}(S, M)}{\Pi^{-}(S, P)} C e l
$$

in which the conclusion determines which term (namely $S$ ) is the Subject (also called the "Minor Term") of the syllogism and which (namely $P$ ) is the Predicate (also called the "Major Term") of the syllogism; the remaining term $M$ is the "Middle Term". We display the Major Premiss as the first premiss and the other premiss (the Minor Premiss) second.

So a deduction is a tree, deducing a proposition (the conclusion) from a multiset of one or more propositions (the assumptions). Some of these deductions are of syllogisms, with two assumptions. Easily one can see that, ignoring the requirement to have a deduction, there are 256 possible such syllogisms; but (less easily) only 24 have deductions, i.e. are the valid syllogisms. Aristotle's Prior Analytics explains which ones are valid and why (we ignore the fact that he ignored 6 of the 24). Rather than re-present his proof theory, and the modern results such as those in von Plato's work, we look at another way of doing it.

§3. Revised formal system. We now consider a revised proof system. We replace the first two rules of Aristotle's system by the following, in natural deduction form:

B1: From $\Pi^{+}(S, P)$ deduce its subaltern $\Sigma^{+}(S, P)$;

B2: From $\Pi^{-}(S, P)$ deduce its subaltern $\Sigma^{-}(S, P)$;

B3: From $\Sigma^{+}(P, S)$ deduce (by conversion) $\Sigma^{+}(S, P)$;

B4: From $\Pi^{-}(P, S)$ deduce (by conversion) $\Pi^{-}(S, P)$.

and keep the two rules of syllogism Bar (for Barbara) and Cel (for Celarent) and the rule of $I P$. Rule $B 4$ is just the old rule $A 2$, renamed. Each of the new rules $(B 1, B 2$ and $B 3)$ is deducible in the old system (without using $B$ ar or $\mathrm{Cel}$, but maybe with the aid of $I P$ ). So every deduction in the new system translates to a deduction in the old system.

Conversely, Aristotle's two basic rules ( $A 1$ and $A 2)$ are deducible using just these four new rules (i.e. without use of syllogisms or $I P$ ): $A 1$ is 
deducible using $B 1$ and $B 3 ; A 2$ is the same as rule $B 4$. We no longer have the nice feature, useful for the proof-theory, that none of the basic rules has an existential premiss.

As before, we say that a direct deduction is one without use of IP. Clearly, anything directly deducible in the old system is directly deducible in the new system. The syllogisms directly deducible in the new system are 11 in number: Bamalip, Barbara, Barbari, Calemes, Calemos, Camestres, Camestros, Celarent, Celaront, Cesare and Cesaro.

CONJeCture 3.1. By using these four basic rules and using a few fixed syllogisms (including Barbara and Celarent), we get a complete IP-free system, i.e. one in which IP is not primitive but admissible.

Before we prove the conjecture, we take a look at some direct proofs in the revised system and a few indirect proofs: it is the latter that we need to make into direct proofs (using whatever extra syllogisms are added).

§4. Examples of Direct Proofs (using the Revised System). We give just three examples:

1. The syllogism "Bamalip":

$$
\frac{\Pi^{+}(M, S) \quad \Pi^{+}(P, M)}{\frac{\Pi^{+}(P, S)}{\Sigma^{+}(P, S)} B 1} \text { Bar }
$$

2. The syllogism "Camestros":

$$
\frac{\frac{\Pi^{-}(S, M)}{\Pi^{-}(M, S)} B 4 \quad \Pi^{+}(P, M)}{\frac{\Pi^{-}(P, S)}{\Pi^{-}(S, P)} B 4}{ }^{\Sigma^{-}(S, P)} B 2
$$

3. The syllogism "Cesaro":

$$
\frac{\frac{\Pi^{-}(P, M)}{\Pi^{-}(M, P)} B 4 \quad \Pi^{+}(S, M)}{\frac{\Pi^{-}(S, P)}{\Sigma^{-}(S, P)} B 2} C e l
$$

Apart from 11 such syllogisms (listed in Section 3), none of the 24 valid two-premiss syllogisms has a direct proof, i.e. all the others only have indirect proofs. In the next section we give four examples. 
§5. Examples of Indirect Proofs. Where an assumption is discharged, we write a label such as $\alpha$ over it and repeat the label as suffix to $I P$ at the place of discharge.

1. The syllogism "Baroco":

$$
\frac{\Pi^{+}(P, M) \frac{\alpha}{\Pi^{+}(S, P)}}{\frac{\Pi^{+}(S, M)}{\Sigma^{-}(S, P)} \Sigma^{-}(S, M)} I P_{\alpha}
$$

2. The syllogism "Bocardo":

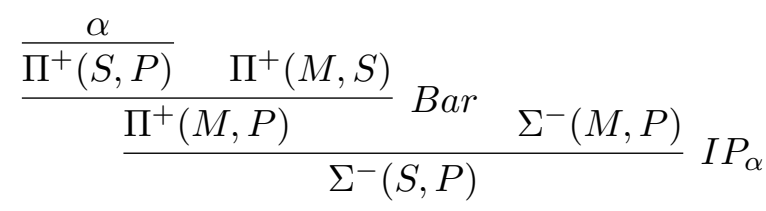

3. The syllogism "Disamis":

$$
\frac{\frac{\alpha}{\Pi^{-}(S, P)} \quad \Pi^{+}(M, S)}{\frac{\Pi^{-}(M, P)}{\Sigma^{+}(S, P)} \text { Cel } \Sigma^{+}(M, P)} I P_{\alpha}
$$

4. The syllogism "Festino":

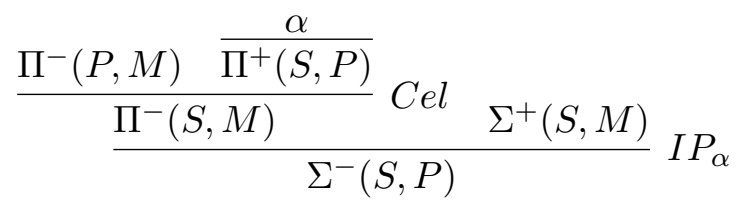

§6. The Extended System. We add, to the four basic rules $B 1, B 2, B 3$ and $B 4$ and the two basic syllogistic rules Bar and $C e l$, the syllogisms Baroco, Bocardo, Disamis and Festino, and call this the Extended System. Despite this name, no rule of indirect proof is included.

Lemma 6.1. If we have a direct deduction of $B$ from $\Gamma, A^{*}$ in the $E x$ tended System, then we may construct one of $A$ from $\Gamma, B^{*}$.

Proof. By induction on the deduction height. The base case is where $A^{*}=B$ and $\Gamma=\emptyset$; so we easily have a zero-height deduction of $A$ from $B^{*}$. For the induction step, we consider the cases:

1. Last step is that, by Rule $B 1$, from $\Pi^{+}(P, Q)$ we deduce $B=$ $\Sigma^{+}(P, Q)$. By inductive hypothesis we can construct a direct deduction of $A$ from $\Gamma,\left(\Pi^{+}(P, Q)\right)^{*}$, i.e. of $A$ from $\Gamma, \Sigma^{-}(P, Q)$. We precede this by deduction, by Rule $B 2$, of $\Sigma^{-}(P, Q)$ from $\Pi^{-}(P, Q)$. This gives us a direct deduction of $A$ from $\Gamma, \Pi^{-}(P, Q)$ as required. 
2. Similar arguments apply to the other three basic rules $B 2, B 3$ and B4.

3. Consider a Barbara step, as in

$$
\begin{array}{cc}
\Gamma^{\prime}, A^{*} & \Gamma^{\prime \prime} \\
\vdots & \vdots \\
\frac{\Pi^{+}(M, P)}{\Pi^{+}(S, P)} & \Pi^{+}(\dot{S}, M) \\
& B a r
\end{array}
$$

where we have wlog assigned the assumption $A^{*}$ to the first premiss. So $B=\Pi^{+}(M, P)$ and $B^{*}=\Sigma^{-}(M, P)$. By the $I H$ (the "Induction Hypothesis"), we may construct a direct deduction of

$$
\begin{gathered}
\Gamma^{\prime}, \Sigma^{-}(M, P) \\
\vdots \\
\dot{A}
\end{gathered}
$$

above which we place a step by Bocardo (and above that the deduction of the second premiss of the Barbara step), as in

$$
\begin{aligned}
& \begin{array}{c}
\Gamma^{\prime \prime} \\
\vdots \\
\Gamma^{\prime}, \quad \frac{\Sigma^{-}(S, P) \quad \Pi^{+}(\dot{S}, M)}{\Sigma^{-}(M, P)} \text { Bocardo }
\end{array} \\
& \vdots
\end{aligned}
$$

giving us, since $B^{*}=\Sigma^{-}(S, P)$, a direct deduction of $A$ from $\Gamma^{\prime}, \Gamma^{\prime \prime}, B^{*}$. If on the other hand $A^{*}$ is in the second premiss of Barbara, we use Baroco instead of Bocardo.

4. Consider a Celarent step, as in

$$
\begin{array}{cc}
\Gamma^{\prime}, A^{*} & \Gamma^{\prime \prime} \\
\vdots & \vdots \\
\frac{\Pi^{-}(M, P)}{\Pi^{-}(S, P)} & \Pi^{+}(\dot{S}, M) \\
& C e l
\end{array}
$$

where we have wlog assigned the assumption $A^{*}$ to the first premiss. By the $I H$, we may construct a direct deduction of

$$
\begin{gathered}
\Gamma^{\prime}, \Sigma^{+}(M, P) \\
\vdots \\
\dot{A}
\end{gathered}
$$


above which we place a step by Disamis (and the deduction of the second premiss of the Celarent step), as in

$$
\begin{gathered}
\Gamma^{\prime \prime} \\
\vdots \\
\Gamma^{\prime}, \quad \frac{\Sigma^{+}(S, P) \quad \Pi^{+}(S, M)}{\Sigma^{+}(M, P)} \text { Disamis } \\
\vdots \\
A
\end{gathered}
$$

giving us, since $B^{*}=\Sigma^{+}(S, P)$, a direct deduction of $A$ from $\Gamma^{\prime}, \Gamma^{\prime \prime}, B^{*}$. If on the other hand $A^{*}$ is in the second premiss of Celarent, we use Festino instead of Disamis.

5. This commits us to having not just Barbara and Celarent in our system of rules, but also Baroco, Bocardo, Festino and Disamis. So we have to consider how to transform one of these. We give just one example: Baroco. Suppose we have

$$
\begin{array}{cc}
\Gamma^{\prime}, A^{*} & \Gamma^{\prime \prime} \\
\vdots & \vdots \\
\frac{\Pi^{+}(\dot{P}, M)}{\Sigma^{-}(S, P)} & \Sigma^{-}(\dot{S}, M) \\
\text { Baroco }
\end{array}
$$

where we have wlog assigned the assumption $A^{*}$ to the first premiss. By the $I H$, we may construct a direct deduction of

$$
\begin{gathered}
\Gamma^{\prime}, \Sigma^{-}(P, M) \\
\vdots \\
\dot{A}
\end{gathered}
$$

above which we place a step by Bocardo (and the deduction of the second premiss of Baroco), as in

$$
\begin{gathered}
\Gamma^{\prime \prime} \\
\vdots \\
\Gamma^{\prime}, \quad \frac{\Sigma^{-}(S, M) \quad \Pi^{+}(S, P)}{\Sigma^{-}(P, M)} \text { Bocardo } \\
\vdots \\
A
\end{gathered}
$$

giving us, since $B^{*}=\Pi^{+}(S, P)$, a direct deduction of $A$ from $\Gamma^{\prime}, \Gamma^{\prime \prime}, B^{*}$. If on the other hand $A^{*}$ is in the second premiss of Baroco, we use Barbara instead of Bocardo.

The result now follows by induction.

The Lemma is one of the Stoic rules of inference, the first thema, T1, for which see [1] or [2]. 
Theorem 6.2. The rule of IP is admissible in the Extended System consisting of the four basic rules B1-B4 and the six basic syllogisms (Barbara, Celarent, Baroco, Bocardo, Disamis and Festino).

Proof. Consider a step by $I P$ with directly deduced premisses:

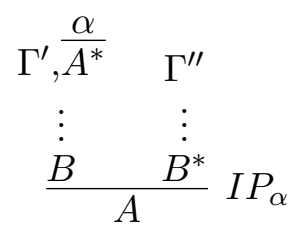

and transform the deduction of the first premiss by the Lemma to a direct deduction of $A$ from $\Gamma^{\prime}, B^{*}$. This may be composed with the deduction of $B^{*}$ from $\Gamma^{\prime \prime}$ to provide a direct deduction of $A$ from $\Gamma^{\prime}, \Gamma^{\prime \prime}$.

Note that it is important for this proof that we refer to Smiley or von Plato's rule of Indirect Proof with its avoidance of multiple or vacuous discharge. For example, if we have also an assumption of $A^{*}$ in the deduction of $B^{*}$, we end up with a direct deduction of $A$ from $\Gamma, \Gamma^{\prime \prime}, A^{*}$-not what is required. That our proof works, however, is not the main reason for preferring this version to that of Corcoran: the better reason is that it seems to be more faithful to Aristotle's use of Indirect Proof.

$\S 7$. Comments. Thus, by changing Aristotle's basic rules slightly and using not just two but six basic syllogisms, we can dispense with his rule of Indirect Proof.

It is of course routine, but tedious, to check this in the case of each of the valid two-premiss syllogisms that is not directly deducible, $13=(24-11)$ in number. But what we have shown applies to any deductions, not just those of the named syllogisms.

Our six basic syllogisms are not the six of the first figure: Baroco and Festino are from the second figure, while Bocardo and Disamis are from the third.

§8. Equivalences and Implications. Do we really need as many as six basic syllogisms? If we define two forms of syllogism to be equivalent if each can be used to deduce the other (using only the four basic rules $B 1 \ldots B 4$ ), and for one to imply the other if the first can be used to deduce (using only the four basic rules $B 1 \ldots B 4$ ) the other (but not conversely), we have the following story.

There are 11 equivalence classes of syllogism. Such a class is fundamental iff it is minimal w.r.t. implication. 6 of these classes are fundamental (we put in bold those syllogisms we happen to have used in our Extended System): Barbara; Baroco; Bocardo; Celarent, Camestres, Calemes 
and Cesare; Disamis, Darii, Dimatis and Datisi; Festino, Ferison, Fresison and Ferio; and 5 classes are non-fundamental: Bamalip and Barbari; Calemos and Camestros; Celaront and Cesaro; Darapti; Felapton and $\mathrm{Fe}$ sapo.

We also have the following nine extra implications (in each case, from a fundamental class to a non-fundamental class, with classes being identified in each case by one representative): Barbara implies Bamalip; Baroco implies Calemos; Bocardo implies Felapton; Celarent implies Calemos; Celarent implies Celaront; Disamis implies Bamalip; Disamis implies Darapti; Festino implies Celaront; Festino implies Felapton.

The 29 implications and many more non-implications implicit in this classification are left as a logical exercise. For example, that Festino implies Felapton is shown by the direct deduction

$$
\frac{\frac{\Pi^{-}(M, P)}{\Pi^{-}(P, M)} B 4 \quad \frac{\Pi^{+}(M, S)}{\Sigma^{+}(S, M)}}{\Sigma^{-}(S, P)} \text { Festino }
$$

and the failure of the converse is shown by the failure of exhaustive search. The drawing of a planar graph to illustrate all these relationships better is left as a non-logical exercise.

Using two syllogisms, $X$ and $Y$ say, gets us (of the 24 valid two-premiss syllogisms) just the syllogisms that $X$ gives us plus those that $Y$ gives us: combining two syllogisms, in other words, will generate three-premiss syllogisms, which are not the ones of primary interest.

So, we cannot do without six basic syllogisms if we aim for completeness without use of IP. But why have we preferred Disamis and Festino to, say, from the first figure, Darii and Ferio (an idea supported by Aristotle)?

§9. Inverses of Syllogisms. We adopt a simple and obvious notation for syllogisms, not intended to suggest that they are propositions rather than rules of inference. $A \rightarrow B \rightarrow C$ is the form of a syllogism with major premiss $A$, minor premiss $B$ and conclusion $C$.

Consider the syllogism: $A \rightarrow B \rightarrow C$; we may invert it in two ways, obtaining (the first way) $C^{*} \rightarrow B \rightarrow A^{*}$ and (the second way) $A \rightarrow C^{*} \rightarrow$ $B^{*}$. The terms may need to be renamed, and the order of the premisses may need to be reversed, to ensure the holding of conventions about where the middle term appears and where the subject and predicate appear.

The two inverses obtained in this way from Barbara, that is:

$$
\Pi^{+}(M, P) \rightarrow \Pi^{+}(S, M) \rightarrow \Pi^{+}(S, P),
$$

are $\Sigma^{-}(M, P) \rightarrow \Pi^{+}(M, S) \rightarrow \Sigma^{-}(S, P)$ and $\Pi^{+}(P, M) \rightarrow \Sigma^{-}(S, M) \rightarrow$ $\Sigma^{-}(S, P)$, known respectively as Bocardo and Baroco. 
The two inverses obtained in this way from Celarent, that is:

$$
\Pi^{-}(M, P) \rightarrow \Pi^{+}(S, M) \rightarrow \Pi^{-}(S, P),
$$

are $\Sigma^{+}(M, P) \rightarrow \Pi^{+}(M, S) \rightarrow \Sigma^{+}(S, P)$ and $\Pi^{-}(P, M) \rightarrow \Sigma^{+}(S, M) \rightarrow$ $\Sigma^{-}(S, P)$, known respectively as Disamis and Festino.

To make this clearer, consider Barbara's first inversion. The first step is to construct $\Pi^{+}(S, P)^{*} \rightarrow \Pi^{+}(S, M) \rightarrow \Pi^{+}(M, P)^{*}$, i.e. $\Sigma^{-}(S, P) \rightarrow$ $\Pi^{+}(S, M) \rightarrow \Sigma^{-}(M, P)$. To conform with nomenclatures in the conclusion, $M$ needs to be renamed as $S$, and vice-versa, as in $\Sigma^{-}(M, P) \rightarrow$ $\Pi^{+}(M, S) \rightarrow \Sigma^{-}(S, P)$, i.e. Bocardo.

The first inversion of Festino first constructs $\Sigma^{-}(S, P)^{*} \rightarrow \Sigma^{+}(S, M) \rightarrow$ $\Pi^{-}(P, M)^{*}$, i.e. $\Pi^{+}(S, P) \rightarrow \Sigma^{+}(S, M) \rightarrow \Sigma^{+}(P, M)$; now $S, M$ and $P$ must be permuted, and we obtain $\Pi^{+}(M, S) \rightarrow \Sigma^{+}(M, P) \rightarrow \Sigma^{+}(S, P)$, but must now reorder the premisses, giving us $\Sigma^{+}(M, P) \rightarrow \Pi^{+}(M, S) \rightarrow$ $\Sigma^{+}(S, P)$, i.e. Disamis.

THEOREM 9.1. The 24 valid syllogisms are in eight inversion groups of 3:

1. (a) The syllogism "Barbara": $\Pi^{+}(M, P) \rightarrow \Pi^{+}(S, M) \rightarrow \Pi^{+}(S, P)$

(b) The syllogism "Bocardo": $\Sigma^{-}(M, P) \rightarrow \Pi^{+}(M, S) \rightarrow \Sigma^{-}(S, P)$

(c) The syllogism "Baroco": $\Pi^{+}(P, M) \rightarrow \Sigma^{-}(S, M) \rightarrow \Sigma^{-}(S, P)$;

2. (a) The syllogism "Celarent": $\Pi^{-}(M, P) \rightarrow \Pi^{+}(S, M) \rightarrow \Pi^{-}(S, P)$

(b) The syllogism "Disamis": $\Sigma^{+}(M, P) \rightarrow \Pi^{+}(M, S) \rightarrow \Sigma^{+}(S, P)$

(c) The syllogism "Festino": $\Pi^{-}(P, M) \rightarrow \Sigma^{+}(S, M) \rightarrow \Sigma^{-}(S, P)$;

3. (a) The syllogism "Calemes": $\Pi^{+}(P, M) \rightarrow \Pi^{-}(M, S) \rightarrow \Pi^{-}(S, P)$

(b) The syllogism "Fresison": $\Pi^{-}(P, M) \rightarrow \Sigma^{+}(M, S) \rightarrow \Sigma^{-}(S, P)$

(c) The syllogism "Dimatis": $\Sigma^{+}(P, M) \rightarrow \Pi^{+}(M, S) \rightarrow \Sigma^{+}(S, P)$;

4. (a) The syllogism "Camestres": $\Pi^{+}(P, M) \rightarrow \Pi^{-}(S, M) \rightarrow \Pi^{-}(S, P)$

(b) The syllogism "Ferison": $\Pi^{-}(M, P) \rightarrow \Sigma^{+}(M, S) \rightarrow \Sigma^{-}(S, P)$

(c) The syllogism "Darii": $\Pi^{+}(M, P) \rightarrow \Sigma^{+}(S, M) \rightarrow \Sigma^{+}(S, P)$;

5. (a) The syllogism "Cesare": $\Pi^{-}(P, M) \rightarrow \Pi^{+}(S, M) \rightarrow \Pi^{-}(S, P)$

(b) The syllogism "Datisi": $\Pi^{+}(M, P) \rightarrow \Sigma^{+}(M, S) \rightarrow \Sigma^{+}(S, P)$

(c) The syllogism "Ferio": $\Pi^{-}(M, P) \rightarrow \Sigma^{+}(S, M) \rightarrow \Sigma^{-}(S, P)$;

6. (a) The syllogism "Bamalip": $\Pi^{+}(P, M) \rightarrow \Pi^{+}(M, S) \rightarrow \Sigma^{+}(S, P)$

(b) The syllogism "Calemos": $\Pi^{+}(P, M) \rightarrow \Pi^{-}(M, S) \rightarrow \Sigma^{-}(S, P)$

(c) The syllogism "Fesapo": $\Pi^{-}(P, M) \rightarrow \Pi^{+}(M, S) \rightarrow \Sigma^{-}(S, P)$;

7. (a) The syllogism "Barbari": $\Pi^{+}(M, P) \rightarrow \Pi^{+}(S, M) \rightarrow \Sigma^{+}(S, P)$

(b) The syllogism "Felapton": $\Pi^{-}(M, P) \rightarrow \Pi^{+}(M, S) \rightarrow \Sigma^{-}(S, P)$

(c) The syllogism "Camestros": $\Pi^{+}(P, M) \rightarrow \Pi^{-}(S, M) \rightarrow \Sigma^{-}(S, P)$;

8. (a) The syllogism "Celaront": $\Pi^{-}(M, P) \rightarrow \Pi^{+}(S, M) \rightarrow \Sigma^{-}(S, P)$

(b) The syllogism "Darapti": $\Pi^{+}(M, P) \rightarrow \Pi^{+}(M, S) \rightarrow \Sigma^{+}(S, P)$

(c) The syllogism "Cesaro": $\Pi^{-}(P, M) \rightarrow \Pi^{+}(S, M) \rightarrow \Sigma^{-}(S, P)$.

In each group, the syllogism with the alphabetically earliest name is listed first, then its first inverse, then its second inverse. 
Proof. By careful hand calculation, as in the examples given. In each case, the first inversion of the syllogism in (a) gives that in (b) and the second gives that in (c). There is no claim that the first inversion of the syllogism in (b) is either always that in (a) or always that in (c). Reordering of premisses interferes: all we claim is that from (b) one inversion leads back to (a) and the other to (c); and similarly from (c).

This is intended to explain why we have chosen, as well as the traditional Barbara and Celarent, the syllogisms Baroco and Bocardo (inverses of Barbara) and the syllogisms Festino and Disamis (inverses of Celarent). We could have abandoned Celarent in favour of one of its three equivalents, such as Calemes; if so, we would have included Dimatis and Fresison (inverses of Calemes). By inspection we can see that, whatever choices we made here, we would still (after inclusion of the inverses) get one member from each of the six basic equivalence classes.

\$10. Related Work. Corcoran [3] gives a different formalisation (or two) of Aristotle's deductive system; but we are unable to see that either is equivalent to von Plato's, or is as faithful to Aristotle. The crucial difference is that Corcoran allows both multiple and vacuous discharge of an assumption; from this it is easy to construct, from the assumptions $\Pi^{+}(M, P)$ and $\Pi^{-}(M, P)$, via Barbara and Celarent, an indirect deduction that $\Sigma^{-}(S, M)$-if all men are pigheaded and no men are pigheaded, then some snake is not a man. This is a correct deduction according to his semantic interpretation. (It is not however a demonstration: the antecedents cannot both be true.) But it doesn't work in [7] or in [13].

Joray [5, 6], correcting and furthering the work of Smith [8], uses ecthesis, the introduction of fresh variables in a fashion similar to that in $\exists$-Elim, to avoid Indirect Proof. For example, his system $\mathbf{S}_{4}$ has no rule of Indirect Proof, but just (i) the syllogisms Barbara, Celarent, Darii and Ferio; (ii) the rules that we have called $A 1, B 2$ and $B 4$; (iii) the rule $I$ Ecth: from $\Sigma^{+}(S, P)$ we may, for a fresh term $Q$, infer both $\Pi^{+}(Q, S)$ and $\Pi^{+}(Q, P)$ (and we'll need to avoid having $Q$ in the conclusion of whatever we are trying to deduce); (iii) the rule $O$-Ecth: from $\Pi^{-}(S, P)$ we may, for a fresh term $Q$, infer both $\Pi^{+}(Q, S)$ and $\Pi^{-}(Q, P)$ (and we'll need to avoid having $Q$ in the conclusion of whatever we are trying to deduce). The missing conversion rule $B 3$ can then be deduced using $A 1$, Darii and I-Ecth. Bocardo may be deduced using Barbara and Ferio (and ecthesis). Baroco may be deduced using Celarent and Ferio (and ecthesis). Joray cites traces of ecthesis in some of Aristotle's arguments.

Tennant [9] is more radical: he abandons all use of syllogisms such as Barbara as primitive and shows how it can all be done in a GentzenPrawitz style natural deduction system (where the role of ecthesis is taken over by $\exists$-Elim and $\forall$-Intro). That is no surprise: the special feature 
however is that no classical rule such as Reductio ad Absurdum is needed and, even better, there are no multiple or vacuous discharges. The whole can then be reformulated in a fragment of his system of Core Logic, where deductions are automatically constructive, normal and relevant. But it is further from Aristotle than a system such as our Extended System or [5].

§11. Conclusion. Much has been written about the 24 valid syllogisms, with mediaeval interest in using names to assist understanding of relationships to the various figures. Our approach is different: to see what syllogisms are needed (on top of four basic rules, not exactly as chosen by Aristotle) to allow avoidance of the rule of Indirect Proof, and what relationships, such as equivalence, implication or inversion, tie them together or separate them. These relationships may be of independent interest.

\section{REFERENCES}

[1] S Bobzien, Stoic syllogistic, Oxford studies in ancient philosophy, vol. 14, Oxford University Press, 1996, pp. 133-192.

[2] S. Bobzien and R. DyCKhofF, Analyticity, balance and non-admissibility of Cut in Stoic logic, Studia Logica, (2018), pp. XX-YY, https://doi.org/10.1007/ s11225-018-9797-5.

[3] J. Corcoran, Completeness of an ancient logic, J. Symbolic Logic, vol. 37 (1972), pp. 696-702.

[4] - A mathematical model of Aristotle's syllogistic, Archiv für Geschichte der Philosophie, vol. 55 (1973), pp. 191-219.

[5] P. JORAY, The principle of contradiction and ecthesis in Aristotle's syllogistic, History and Philosophy of Logic, vol. 35 (2014), pp. 219-236.

[6] - A completed system for Robin Smith's incomplete ecthetic syllogistic, Notre Dame Journal of Formal Logic, vol. 58 (2017), pp. 329-342.

[7] T. J. Smiley, What is a syllogism?, J. Philosophical Logic, vol. 2 (1973), pp. $136-154$.

[8] R. Smith, Completeness of an ecthetic syllogistic, Notre Dame Journal of Formal Logic, vol. 24 (1983), pp. 224-232.

[9] N. Tennant, Aristotle's syllogistic and Core logic, History and Philosophy of Logic, vol. 35 (2014), pp. 120-147.

[10] A. S. Troelstra and H. Schwichtenberg, Basic proof theory, Cambridge University Press, 1996 and 2001.

[11] J. VON PLATO, The great formal machinery works: Theories of deduction and computation at the origins of the digital age, Princeton University Press, 2011.

$[12]-$, Logical reasoning: First principles and deductive machinery, Cambridge University Press, 2011.

[13] - Aristotle's deductive logic: a proof-theoretical study, Concepts of proof in mathematics, philosophy and computer science (D. Probst and P. Schuster, editors), De Gruyter, 2016, pp. 323-346.

UNIVERSITY OF ST ANDREWS, UK

E-mail: roy.dyckhoff@gmail.com 